\title{
Development of Cable Dielectric Loss Tester
}

\author{
Zheng Wendong \\ DaGu south Road No. 1310 Hexi District Tianjin China \\ Tianjin Key Laboratory of Information Sensing and \\ Intelligent Control; \\ Tianjin, China \\ e-mail: 1294891558@qq.com
}

\author{
$\mathrm{Su}$ Xin \\ Hongqi Road No. 278, Nankai District, Tianjin, China \\ Chengxi Power Supply Bureau, Tianjin Power Grid \\ Tianjin, China \\ e-mail: susin@126.com
}

\begin{abstract}
Insulation dielectric loss of power cable is more obvious in ultra-low frequency than in other frequency band. The insulation dielectric loss effectively describes power cable insulating properties and the DC characteristics of the medium. A new dielectric loss tester is designed, using quasi-synchronous harmonic analysis method to measure the coefficient of dielectric loss in low frequency of $0.1 \mathrm{~Hz}, 1 \mathrm{~Hz}$ and $10 \mathrm{~Hz}$. By comparing the differences between the coefficient of dielectric loss, the characteristics of cable insulation dielectric loss can be approximately described.
\end{abstract}

Keywords-cable; tester; dielectric loss; ultra-low frequency

\section{INTRODUCTION}

The insulation characteristic of power cable dielectric can be effectively and accurately reflected by tangent value of the dielectric loss angle. Dielectric loss can cause dielectric internal heat loss, which result in power loss that is proportional to the frequency and voltage [1]. The cable's insulation dielectric loss, therefore, is directly related to the cable transmission capacity and service life of cable. In this case, it is necessary to study the dielectric loss. By measuring tangent value of dielectric loss angle, it can effectively detect some latent defect of cable insulation performance and timely find the hidden accident [2-3]. At the same time, it is helpful to maintaining power cable safe running and improving the reliability of power transmission line.

Water-tree in cable insulation dielectric will continue to grow and expand in the environment of long-term moist when the cable works. Water-tree makes the breakdown of running power cable, which leads to a large area power outages and some power accident. Through the study it is found that samples of XLPE power cable containing water-tree is completely different from normal samples in some insulating properties such as water-tree rectification effect, conductive effect, harmonic effect, etc. AC voltage signal of ultra-low frequency is used to test dielectric loss [4-6]. Not only does it keep the DC method signal characteristics obviously and have the outstanding ability to resist the power frequency interference, but also result in high sensitivity detection of water tree deterioration and elimination one-way stray current effect in the AC case.

\author{
Yang Genghuang \\ Dagu south Road No.1310 Hexi District Tianjin China \\ Tianjin Key Laboratory of Information Sensing and \\ Intelligent Control; \\ Tianjin, China \\ e-mail: ygenghuang@126.com \\ Cao Mingzhe \\ Dagu south Road No.1310 Hexi District Tianjin China \\ Tianjin Key Laboratory of Information Sensing and \\ Intelligent Control; \\ Tianjin, China \\ e-mail: 849738243@qq.com
}

Dielectric loss test carried out in the ultra-low frequency AC voltage has advantages, compared with the traditional measuring method under the voltage in power frequency [7]. The test improves the measurement accuracy and reliability.

\section{SYSTEM STRUCTURE}

The digital dielectric loss instrument is based on microprocessor. In the voltage of ultra-low frequency, dielectric loss is measured. The system mainly consists of high-voltage step-up transformer, rectifier circuit, inverter circuit, signal acquisition circuit, signal amplification circuit, filter circuit, protection circuit, micro-controller, man-machine interface and other parts, the system structure is shown in Fig. 1 .
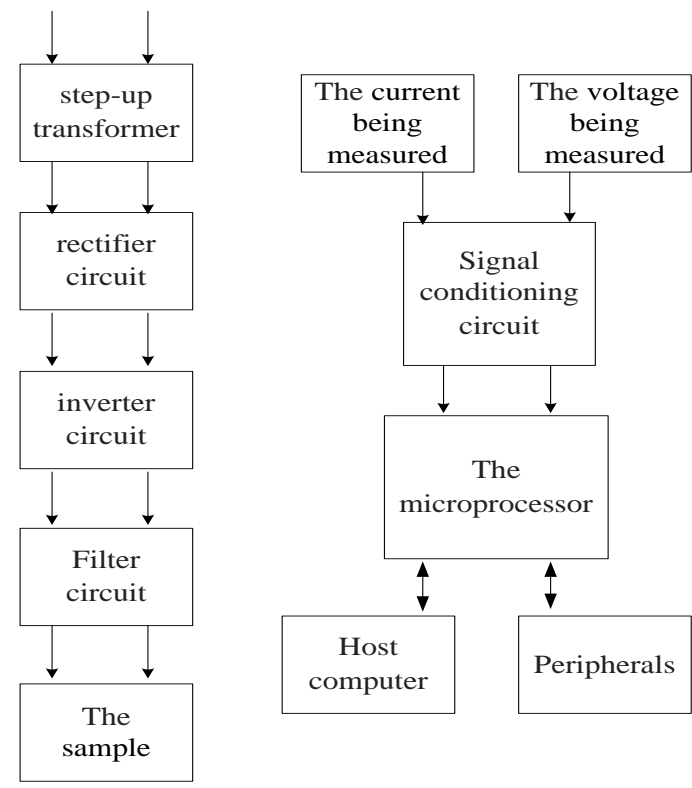

Figure 1. The system's overall design

\section{MEASUREMENT OF TANA IN 0.1HZ FREQUENCY}

Currently, the traditional mass loss tester is under conditions of power frequency to be measured by using the 
bridge, the most commonly used bridge is Schering Bridge [8]. Bridge method cannot test high voltage large capacity samples. In the case of ultra-low frequency, the commonly used bridge can't balance, which often lead to some considerable error.

The system uses the quasi -synchronization harmonic analysis method, it has higher anti-interference ability. This method that utilizes the unique advantages of the FFT in the small signal analysis and adopts the research achievements in the field of digital signal processing (DSP), combined with the quasi synchronous algorithm, eliminate all the external disturbance and do some special analysis for the waveform of voltage and current that is the signal of weak current flowing from the samples of dielectric power cable, to obtain the phase and amplitude of fundamental component of the voltage and current signal and thus to obtain a dielectric loss angle tangent value of the cable tested.

\section{HARDWARE DESIGN}

Hardware circuit part of the measurement system includes the following several sections: rectifier circuit, inverter circuit, filter circuit, signal acquisition and regulate circuit, anti-breakdown protection circuit, microprocessor control section, etc.

\section{A. Power supply}

The power supply requires output amplitude for $2 \mathrm{kV}$ and frequency of $0.1 \mathrm{~Hz}$ sinusoidal $\mathrm{AC}$ signal. It is that $220 \mathrm{~V} / 50 \mathrm{~Hz}$ use voltage booster and AC - DC - AC inverter technology to ultimately control the amplitude and frequency.

Compared with the AC - AC inverter circuit, the main advantage of $\mathrm{AC}$ - $\mathrm{DC}$ - $\mathrm{AC}$ inverter circuit is that the frequency of the output sine wave is no longer limited by the input power frequency. The upper frequency limit of $\mathrm{AC}$ - AC inverter is about $1 / 3$ to $1 / 2$ of the power frequency, which can't meet the design requirements. The system adopts the design of AC - DC - AC inverter technology can meet the requirements of ultra-low frequency power supply. The $220 \mathrm{~V} / 50 \mathrm{~Hz}$ voltage through the step-up transformer, and then through the rectifier bridge, and then through the integral circuit outputs a small fluctuations AC voltage [9-10]. And then through the inverter bridge circuit composed of IGBT, it outputs a voltage of $2 \mathrm{kV}$ and frequency of $0.1 \mathrm{~Hz}$ sinusoidal AC. The principle of the inverter circuit is shown in Fig.2.

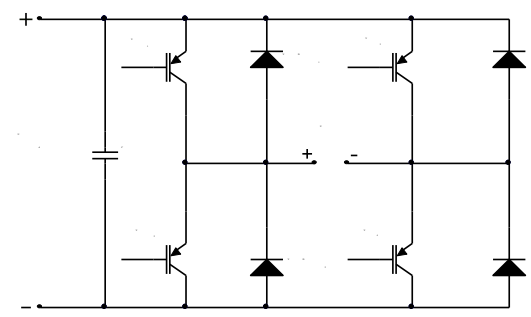

Figure 2. The principle of the inverter circuit

\section{B. Signal acquisition circuit}

Because voltage transformer and current transformer itself can introduce phase difference, seriously influence the accuracy of the measurement, so the signal acquisition use the form of resistor divider to collect voltage signal and current signal. Signal acquisition circuit of the system is shown in Fig .3, Where $\mathrm{Zx}$ is the capacitance dielectric sample that will be measured, R2 and R3 is lossless standard resistor.

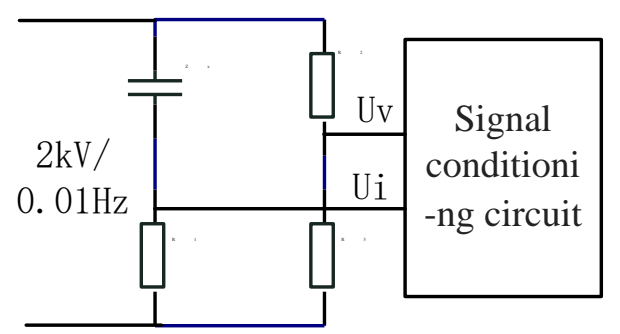

Figure 3. Signal acquisition circuit of the system

The sampling resistor $\mathrm{R} 1$ in series with the $\mathrm{Zx}$ is used to detect a current signal flowing through the test sample, and the test voltage is obtained by the partial pressure through the resistors R2 and R3. After using the signal conditioning circuit to analysis and process voltage and current signals, the results is processed by a digital signal processor. At last through microprocessor operation you can get the value of the dielectric loss angle tangent.

\section{Signal amplification circuit}

In this ultra-low frequency test system, the amplitude of the acquisition current signal is generally small. This signal can't be processed, otherwise it is susceptible to interfered from outside signals. If the acquired current is directly amplified and A/D converted, then the interfering signal can't meet requirements of sampling theorem when the signal is sampled, resulting in frequency aliasing. In order to improve the SNR of the signal, the signal must be low-pass filtered before the signal is amplified. The system structure of Weak current amplifier circuit is shown in

Fig .4.

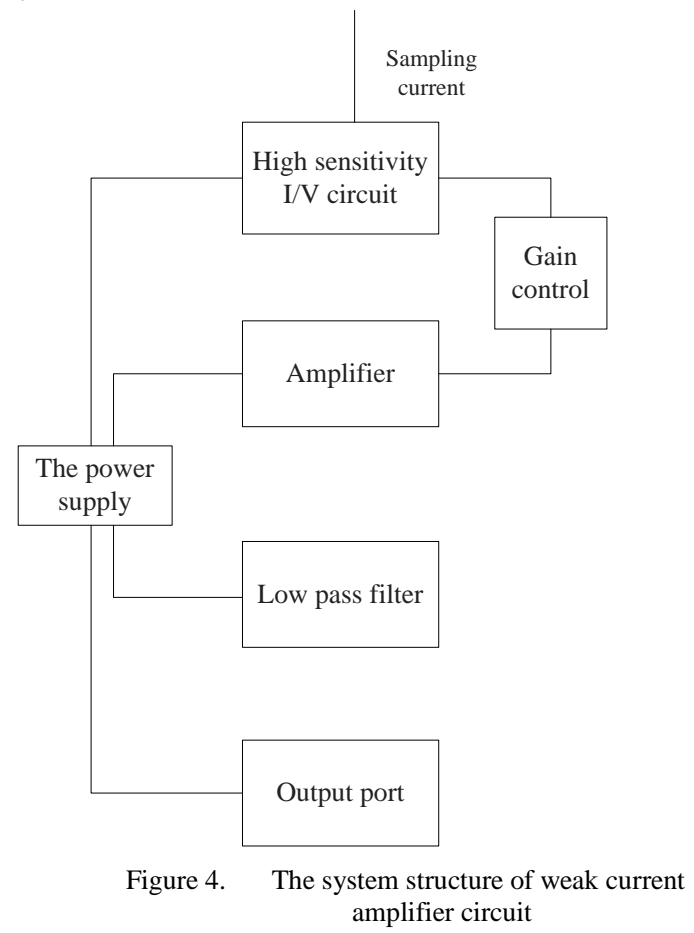




\section{D. the prevent breakdown protection circuit}

As the life and the working environment of measured cable XLPE are different, the insulation deterioration, partial discharge, and other Defects in these equipments are also different. Under high voltage, when the dielectric loss of the cable is serious, then the tested Insulating medium may be breakdown. Therefore, it will damage equipments, but also results in accidents of electric power. So it is necessary to take protective measures for this system. In the case of the comprehensive factors, choosing TVS(Transient suppression diode), the circuit system is Fig .7.

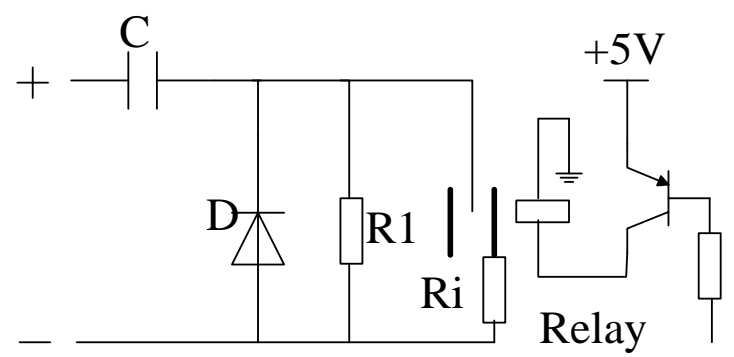

Figure 5. Protection circuit of the system

\section{QUASI SYNCHRONIZATION HARMONIC ANALYSIS METHOD}

The biggest advantage of Quasi-synchronous sampling principle is to eliminate synchronization by adding sampling points of a cycle and increasing the sampling period. At the same time, the corresponding algorithm is used to do data analysis and processing, which can effectively solve the problem that non-integer period sampling affect accuracy of harmonics analysis.

\section{A. Quasi synchronous algorithm}

Harmonic analysis is based on the synchronization algorithm that is based on complete sampling period. In the process of measurement, there is much interference of various factor, it is very difficult to sample the fundamental wave signal over each complete period. Synchronization algorithm can make the measurement error is large, therefore, it must use other suitable algorithm. Quasi-synchronization algorithm is a better algorithm which is widely used. When sampling periodic error is small, the use of quasi-synchronization algorithm can improve average estimation precision of the periodic function. Data processing of quasi synchronous algorithm are as follows: First of all, the signal is sampled for limited times (The diagram is an example that analyze three sampling cycles sample). Since it is a periodic signal, so the signal will be processed $\mathrm{N}$ times. According to determine the algorithm calculates processed voltage for the first time, and then, the calculation will always be do in turn until the last remaining one voltage waveform. As can be seen from the above data processing, the original data are only three cycles, $3 \mathrm{~N}$ times cycles are obtained by quasi synchronous algorithm, which makes the collected data can be fully utilized. Quasi-synchronous sampling principle is shown in Fig .4.

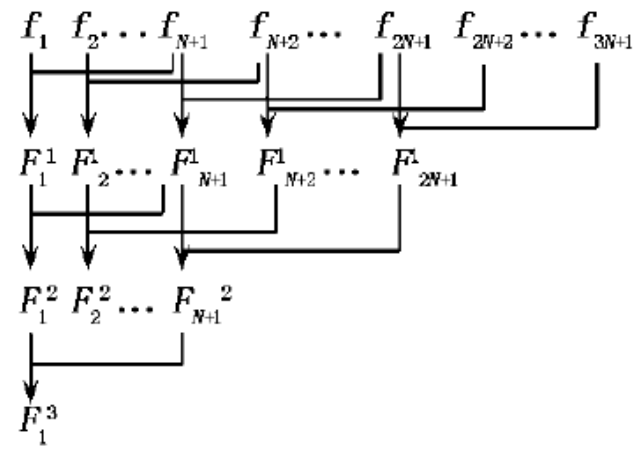

Figure 6. Quasi-synchronous sampling principle

\section{B. Harmonic analysis method}

Based on some of the latest research results and compared to the existing some dielectric loss measuring instruments, it is found that quasi-synchronization harmonic wave analysis method can effectively resist high order clutter interference that can lead to some error or even wrong. Harmonic wave analysis method is that data is FFT transformed to extract the fundamental component. Then according to the formula, the fundamental factor can be calculated. The result is obtained by the corresponding data processing. In the process of harmonic wave analysis, the voltage and the current signals are sampled at the same time in equal time interval period. The testing voltage and current data are stored in the microprocessor. Using fourier transfer achieve the purpose of the harmonic decomposition. By decomposition results of fourier transform, we can calculate the phase difference between the fundamental component of the voltage and the current. Finally, based on the phase difference, the use of tangent calculation formula can get dielectric loss Angle tangent value.

\section{CONCLUSION}

On the study of the dielectric characteristics of the sample, we conducted experiments under $2 \mathrm{kV} / 50 \mathrm{~Hz}$, $2 \mathrm{kV} / 10 \mathrm{~Hz}, 2 \mathrm{kV} / 1 \mathrm{~Hz}, 2 \mathrm{kV} / 0.1 \mathrm{~Hz}$ conditions. The test results indicate that for new XLPE cables, regardless of its size of the frequency, its tan $\delta$ shows no remarkable change, while for defective cross linking polyethylene cable, $\tan \delta$ increases as the frequency decreases. Thus for this method, the aging is easy to detect, $\tan \delta$ measured at ultra-low frequency can effectively reflects DC properties of the cable insulation layer.

The smaller dielectric loss tan $\delta$ or leakage current is, the better the insulating properties of dielectric is. According to the relevant regulations, $\tan \delta$ value or current value of good insulation dielectric should not exceed the certain value or a specific range. In an actual dielectric insulation performance testing process, it is necessary to compare the dielectric loss tangent value or the current value flowing through the dielectric measured and average value or a specific range measured in repeated experiment. According to the results of comparison, the insulation can be effectively judged. If difference of the comparison result is obvious or very large, even if the dielectric loss tangent value and the current value does not exceed the specified standard, the insulating dielectric is also considered unqualified dielectric. 


\section{REFERENCES}

[1] Shu yan Yang, Fan Yang, etc. $0.1 \mathrm{~Hz}$ ultra-low frequency sine wave pressure test technology and application [J]. High voltage technology, 2004.30:79-80

[2] Lei Gao $.0 .1 \mathrm{~Hz}$ ultra-low frequency sine wave voltage test system design[D]. Southwest Jiaotong University master's degree thesis, 2010

[3] Xiao-quan Zheng, guo-hong wang. ultra-low frequency loss characteristics research of XPLE insulation cable containing Water-tree[J].Insulation material communications, 2000.04 -0030-04.

[4] Yonghe Li . Variable voltage variable frequency power supply design based on DSP[D]. Shanghai jiaotong university Master degree thesis, 2013:30-36

[5] Hui Cheng . Developed dielectric loss factor measurement system based on quasi-synchronization algorithm[D].Harbin Polytechnic University master's degree thesis, 2007,15-30.

[6] Yuanfang Wen. High voltage technology [M]. Wuhan: huazhong university of science and technology press, 2001:113-129.
[7] Zhijie wang, Yufa Xu, Sanming Liu etc. Electric power equipment state monitoring and fault diagnosis[M]. Shanghai: Shanghai jiaotong university press, 2012:80-99.

[8] Changchang Wang, Fuqi Li, Shengyou Gao. Online monitoring and fault diagnosis of power equipment[M]. Beijing: tsinghua university press, 2006:66-75.

[9] Hongqi Jiang. The research and development of the digital dielectric loss measuring instrument[D]. Shenyang university of technology master's degree thesis.2013:29-38.

[10] Siwei Qiu. Design and implementation of dielectric los measurement instrument[D]. University of electronic science and technology master's degree thesis.2012:24-57.

[11] Yu Zhao. The dielectric loss test system based on SOPC technology research[D]. Shanghai jiaotong university Master degree thesis, 2013:16-23.

[12] Changjian Si. Measurement of dielectricloss factor of high voltage capacitive apparatus base on DSP[D]. Harbin Polytechnic University master's degree thesis, 2010:26-35 